\title{
Impacto de um bundle nas taxas de pneumonia associada à ventilação mecânica (PAV) em uma unidade de terapia intensiva pediátrica em Londrina-PR
}

\section{Impact of the ventilator bundle on ventilator-associated pneumonia (VAP) rates in a pediatric intensive care unit in Londrina-PR}

\author{
Marcia Regina Eches Perugini ${ }^{1}$ Vitor Hugo Perugini ${ }^{2}$; Fernanda Dias Figueira ${ }^{3}$, Louise \\ Marina Silva Fontana ${ }^{4}$, Juliana Jose Diniz ${ }^{5}$, Daniela Lucio Dos Santos ${ }^{6}$, Renata Aparecida \\ Belei $^{7}$, Eliana Carolina Vespero ${ }^{8}$, Marsileni Pelisson ${ }^{9}$, Aline Tancler Stipp-Abe ${ }^{10}$ Jaqueline \\ Dario Capobiango ${ }^{11}$
}

\begin{abstract}
Resumo
Infecções associadas aos cuidados de saúde (IRAS) constituem importante preocupação à segurança do paciente ao redor do mundo. Pneumonia associada à ventilação mecânica (VAP) é a principal causa de morte entre IRAS, com mortalidade de 15 a 70\%, dependendo da população de pacientes. O Centro de Controle e Prevenção de Doenças (CDC) recomendou que todas as UTIs implementassem um bundle de para reduzir a taxa de PAV. Os objetivos do presente estudo foram analisar o efeito do pacote de intervenções de controle da infecção, a educação, as taxas de PAV na Unidade Pediátrica de Terapia Intensiva (UTIP) do Hospital Universitário de Londrina-PR. Este estudo foi realizado entre janeiro e dezembro de 2013 e consistiu em três períodos: pré-intervenção, intervenção e pós-intervenção. A intervenção educativa foi dada a 86 trabalhadores da saúde (PS), e um questionário também foi realizado no pré e pós-intervenção. No geral, foram avaliadas 135 oportunidades de atendimento ao paciente. A higiene das mãos diminuiu do período pré para o pós-intervenção, entretanto a intubação gástrica por via oral, a manutenção da cabeceira da cama entre $30-45^{\circ}$, a pressão do cuff endotraqueal e remoção de condensação do circuito respirador aumentaram significativamente do período pré para o pós-intervenção. A taxa de PAV foi $49,6 \%$ durante o período de pré-intervenção e 17,5\% durante o período pós-intervenção demonstrando uma redução de $64,8 \%$. Nossos resultados mostram que a implementação do pacote de intervenções de controlo da infecção foi associada com uma redução significativa na taxa de VAP.
\end{abstract}

Palavras-chave: Pneumonia associada à ventilação. Bundle. Prevenção. PAV.

\footnotetext{
Abstract

Healthcare-associated infections (HAI) are an important patient safety concern around the globe. Ventilatorassociated pneumonia (VAP) is the leading cause of death among HAI, with attributable mortality ranging from 15 to $70 \%$ depending on the patient population. The Center for Disease Control and Prevention (CDC)

${ }^{1}$ Graduação em Bachaerlado em Ciências Biológicas- Modalidade Médica e Doutora em Doenças Infecciosas e Parasitárias pela Universidade São Paulo.

${ }^{2}$ Discente do curso de Medicina pela Universidade Estadual de Londrina, Brasil.

${ }^{3}$ Graduação em Farmácia pela Universidade Estadual de Londrina, Brasil.

${ }^{4}$ Graduação em Enfermagem pela Universidade Estadual de Londrina, Brasil.

${ }^{5}$ Graduação em Medicina. Especialização em Controle de Infecção Hospitalar e em Serv. Saúde pela Universidade Estadual de Londrina, Brasil.

${ }^{6}$ Graduação em Medicina. Residência médica pela Universidade Estadual de Londrina, Brasil.

${ }^{7}$ Graduação em Enfermagem pela Universidade Estadual de Londrina, Brasil. Doutorado em Educação Área de Ensino na Educação Brasileira pela Universidade Estadual Paulista Júlio de Mesquita Filho.

${ }^{8}$ Graduação em Farmácia e Doutorado em Microbiologia pela Universidade Estadual de Londrina, Brasil.

${ }^{9}$ Graduação em Farmácia e Mestrado em Microbiologia pela Universidade Estadual de Londrina, Brasil.

${ }^{10}$ Graduação em Farmácia pela Universidade do Sagrado Coração. Doutoranda em Microbiologia na Universidade Estadual de Londrina.

${ }^{11}$ Graduação em Medicina e Doutora em Ciências da Saúde pela Universidade Estadual de Londrina.

${ }^{11}$ Graduada em Medicina pela UEL, doutora em Ciências da Saúde pela UEL. Docente adjunta do departamento de Pediatria do CCS/UEL.
} 
has recommended that all ICUs implement a ventilator bundle to reduce the VAP rate. The purposes of the present study were to examine the effect of the bundle of infection control interventions, education, VAP rates in the Pediatric Intensive Care Unit (PICU) of the Hospital Universitário of Londrina-PR. This study was conducted between January and December 2013 and consisted of three periods: pre-intervention, intervention and post-intervention. An educational intervention was given to 86 healthcare workers (HCWs) about bundles to prevent VAP, and a questionnaire was also performed pre and post-intervention. Overall, 135 opportunities of patient care were evaluated. The compliance with hand hygiene and the use of gloves and gowns did not improve, but orotracheal intubation, maintenance of the patients in a $30-45^{\circ}$ head of bed elevation, endotracheal cuff pressure and removal of condensate from ventilator circuts increased significantly when comparing pre- and post-intervention. The VAP rate was $49.6 \%$ during the pre-intervention period and $17.5 \%$ during the post-intervention period demonstrating a $64.8 \%$ reduction in VAP rate. Our results show that implementation of the bundle of infection control interventions was associated with a significant reduction in VAP rate.

Keywords: Ventilator-associated pneumonia. Bundle. Prevention. VAP.

\section{Introdução}

Pneumonia associada à ventilação mecânica (PAV), definida como infecção pulmonar diagnosticada após 48 horas de ventilação mecânica, é a segunda causa mais comum de infecção relacionada à assistência à saúde, e acarreta aumento de morbidade e mortalidade, além da elevação dos custos hospitalares (AGÊNCIA NACIONAL DE VIGILÂNCIA SANITÁRIA, 2013; BABCOCK et al., 2004; HORAN; ANDRUS; DUDECK, 2008; ROSENTHAL et al., 2012).

Diversos fatores podem favorecer $o$ desenvolvimento da infecção pulmonar associada a ventilação, entre eles, a contaminação do tubo endotraqueal, do aparelho umidificador, dos cateteres intravenosos ou das mãos dos profissionais de saúde (MORINEC, JACOBONI, MACNETT, 2012). A intubação endotraqueal é seguramente o fator mais importante, no entanto, outros fatores como internação em unidade de cuidados intensivos, utilização de antibióticos, doenças de base, colonização da nasofaringe, refluxo gastrointestinal, sonda nasogástrica e aspiração pelas vias aéreas, devem ser considerados (AGÊNCIA NACIONAL DE VIGILÂNCIA SANITÁRIA, 2013; KOLLEF, 2011; LAWRENCE, FULBROOK, 2011; PRINCIPI; ESPOSITO, 2007).

O diagnóstico preciso de PAV ainda não é simples, pois depende de critérios clínicos, radiológicos e laboratoriais, pode sofrer interferência das condições do próprio paciente (CURLEY; SCHALENSTOCKER, 2006).

De acordo com o Centro de Controle de Doenças de Atlanta (CENTER OF DISEASE CONTROL AND PREVENTION, 2003) os critérios de diagnóstico de PAV em crianças são: 2 ou mais Raio X seriados com um dos seguintes achados: infiltrado persistente, consolidação, cavitação ou pneumatocele e pelo menos, três dos parâmetros: piora da troca gasosa e pelo menos três dos seguintes: instabilidade térmica sem outra causa aparente; leucopenia ou leucocitose e desvio a esquerda; aumento de secreção purulenta ou alteração em sua característica ou aumento da secreção pulmonar ou da necessidade de aspiração; apnéia, taquipnéia, batimento de asas nasais com retração dos arcos costais, gemência; sibilos, crepitações ou roncos; tosse; bradicardia ou taquicardia (HORAN; ANDRUS; DUDECK, 2008) Os critérios nacionais de classificação de PAV são semelhantes aos do CDC, exceto pela presença de taquicardia e bradicardia (AGÊNCIA NACIONAL DE VIGILÂNCIA SANITÁRIA, 2013).

Um pacote de medidas para prevenção de $\mathrm{PAV}$, atualmente denominadas bundles, foi proposto pelo CDC (CENTER FOR DISEASE CONTROL AND PREVENTION, 2003), e readequado posteriormente para crianças (O' KEEFE-MC- 
CARTHY ET; SANTIAGO; LAU, 2008). Tais medidas incluem elevação da cabeceira do leito entre 15 a 30 graus, para recém-nascidos, e 30 a 45 graus, para crianças maiores; pausa diária da sedação e avaliação de prontidão para extubação: profilaxia da úlcera péptica e de trombose venosa profunda; higiene oral com clorexidina e circuito de ventilação livre de condensação.

Assim, em virtude das elevadas taxas de PAV em nosso serviço, este estudo tem como objetivo implantar o bundle de PAV, verificar a frequência de pneumonia em crianças submetidas a ventilação mecânica, assim como avaliar a adesão da equipe de saúde às medidas preventivas recomendadas, antes e após o treinamento.

\section{Materiais e Métodos}

Trata-se de um estudo transversal, de intervenção realizado na Unidade de Terapia Intensiva Pediátrica (UTP) do Hospital Universitário de Londrina (HU), um hospital terciário que possui 317 leitos, sendo 15 leitos destinados ao Pronto Socorro Pediátrico, 34 leitos à Enfermaria Pediátrica, 5 à Unidade de Terapia Intensiva Pediátrica.

Nas fases pré e pós-intervenção foram avaliadas as taxas de PAV nas crianças que se encontravam internadas na UTP com diagnóstico de PAV por, pelo menos, 48 horas, durante um período de seis meses.

Foram realizadas, ainda, observações de toda a equipe assistencial, dividida em quatro categorias: médicos e residentes da pediatria, fisioterapeutas e residentes da fisioterapia, enfermeiros, residentes e auxiliares de enfermagem. Cada categoria teve definidas suas atribuições para prevenção de PAV. Cada profissional foi observado em, pelo menos 15 oportunidades de contato com os pacientes e, o número mínimo de observações estipulado para cada profissional foi 15 .

Foram avaliadas as oportunidades de adoção de medidas de prevenção de pneumonia por um observador anônimo, devidamente treinado, que realizou visitas à UTP nos turnos matutino, vespertino e noturno. O observador preencheu um check list referente aos bundles com os dados referentes a cada observação, tendo permanecido no ambiente por no máximo 30 minutos. As observações foram realizadas em sigilo, com o conhecimento apenas das chefias para evitar alterações de comportamento dos profissionais que prestavam assistência, que pudessem interferir na pesquisa.

Os quesitos avaliados no check list deste estudo foram a higiene das mãos, antes e após contato com paciente ou equipamento, elevação da cabeceira (entre $30^{\circ} \mathrm{e} 45^{\circ}$ ), sonda gástrica por via oral, higiene oral (três vezes ao dia, uma em cada período), circuito adequado (sem sujidades, danos ou acúmulo de líquidos) e insuflação do CuffI.

$\mathrm{Na}$ fase de intervenção, foi realizado um treinamento da equipe assistencial através de aula expositiva dialogada. Foram abordadas as medidas que fazem parte do bundle de prevenção de PAV. Para avaliar o nível de conhecimento sobre tais medidas foi aplicado um pré e, um pós-teste. A equipe foi dividida em várias turmas para realização do treinamento com carga horária de 4 horas, por um período de 60 dias com aulas para que todos os profissionais tivessem a oportunidade de participar. A seguir foram elaborados cartazes e de folders na unidade para reforçar os conteúdos discutidos nas aulas.

Os dados das observações foram analisados e comparados pelo programa EpiInfo, versão 3.4.3. de novembro de 2007.

O presente trabalho foi aprovado pelo Comitê de Ética em Pesquisa envolvendo seres humanos da Universidade Estadual de Londrina.

\section{Resultados}

O treinamento foi ministrado para 86 profissionais da equipe de saúde da UTP, entre eles: médicos, 
fisioterapeutas, enfermeiros, auxiliares de enfermagem e residentes. $\mathrm{Na}$ tabela 1 pode ser verificada a frequência de adesão às medidas de precaução de PAV nos períodos pré e pós-intervenção, por categoria profissional.

Higiene das mãos foi realizada em $61 \%$ de 49 oportunidades da fase pré-intervenção, porém com redução para $25 \%$ de 86 oportunidades na fase pósintervenção. Os profissionais em que mais se verificou aumento na higiene das mãos da fase pré para a pósintervenção foram os fisioterapeutas, 86,6\% (13/15) para $100 \%(8 / 8)$ e auxiliares de enfermagem de $21 \%(4 / 19)$ da fase pré para $40 \%(8 / 20)$ na fase pósintervenção.

O item com maior adesão compreendeu a manutenção da cabeceira elevada entre $30^{\circ}$ e $45^{\circ}$ com 77\% (646/833) na fase pré-intervenção e 97\% (662/676) na fase pós, em todas as categorias profissionais. Da mesma forma a utilização de sonda gástrica via oral aumentou de 56,5\% (403/449) antes para 96,8\% (484/480) após treinamento e o Cuff se manteve insuflado em 52\% (59/113) na fase pré e aumentou para $73 \%(19 / 26)$ na pós. O Trach care apresentou-se em boas condições em percentual elevado, tanto na fase pré 100\% (93/93) quanto na pósintervenção 99\% (106/107).

Gráfico 1 - Comparação do percentual de adesão às medidas recomendadas pelo bundle de prevenção de Pneumonia Associada à Ventilação Mecânica (PAV) pela equipe de saúde da UTP do HU de Londrina, nas fases pré e pós-intervenção educacional.

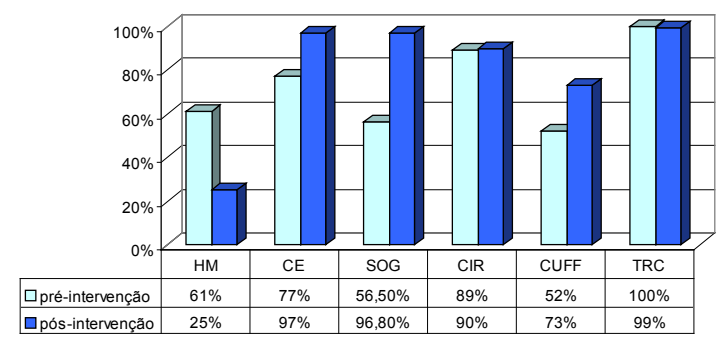

HM- higiene das mãos antes e após contato; CE- cabeceira elevada; SOG- sonda gástrica por via oral; CIR- circuito respirador limpo; CUFF-Cuff insuflado; TRC- Track care em boas condições.

Fonte: autores

Tabela 1 - Percentual de adesão às medidas do bundle para prevenção de PAV antes e após o treinamento da equipe assistencial da UTP do HU de Londrina, por categoria profissional.

\begin{tabular}{|c|c|c|c|c|c|c|c|c|}
\hline & & HM & $\mathbf{C E}$ & SGO & CIR & CUFF & TRC & $\begin{array}{l}\text { Valor } \\
\text { de } p\end{array}$ \\
\hline \multirow{2}{*}{$\begin{array}{l}\text { Auxiliares de } \\
\text { Enfermagem }\end{array}$} & pré & $\begin{array}{c}\mathbf{2 1 \%} \\
(4 / 19)\end{array}$ & $\begin{array}{c}\mathbf{7 1 , 8 \%} \\
(79 / 110)\end{array}$ & $\begin{array}{c}\mathbf{5 7 , 3 \%} \\
(59 / 103)\end{array}$ & $\begin{array}{c}\mathbf{8 7 , 1 \%} \\
(95 / 109)\end{array}$ & $\begin{array}{l}\mathbf{4 6 , 6 \%} \\
(14 / 30)\end{array}$ & $\begin{array}{l}\mathbf{1 0 0 \%} \\
(24 / 24)\end{array}$ & \multirow{2}{*}{0,0017} \\
\hline & pós & $\begin{array}{l}\mathbf{4 0} \% \\
(8 / 20)\end{array}$ & $\begin{array}{c}\mathbf{9 8 , 2 \%} \\
(106 / 108)\end{array}$ & $\begin{array}{c}\mathbf{9 6 , 3 \%} \\
(104 / 108)\end{array}$ & $\begin{array}{l}\mathbf{9 0 , 7 \%} \\
(98 / 108)\end{array}$ & $\begin{array}{c}\mathbf{7 1 , 4} \% \\
(5 / 7)\end{array}$ & $\begin{array}{c}96 \% \\
(24 / 25)\end{array}$ & \\
\hline \multirow{2}{*}{ Enfermeiros } & pré & $\begin{array}{c}\mathbf{5 7 , 1 \%} \\
(4 / 7)\end{array}$ & $\begin{array}{c}\mathbf{7 2 , 2} \% \\
(135 / 187)\end{array}$ & $\begin{array}{c}\mathbf{5 6 , 3 \%} \\
(98 / 174)\end{array}$ & $\begin{array}{c}\mathbf{8 9 , 2 \%} \\
(166 / 186)\end{array}$ & $\begin{array}{c}\mathbf{4 9 \%} \\
(23 / 47)\end{array}$ & $\begin{array}{l}\mathbf{1 0 0 \%} \\
(39 / 39)\end{array}$ & \multirow[b]{2}{*}{0,0027} \\
\hline & pós & $\begin{array}{c}\mathbf{4 2 , 8 \%} \\
(3 / 7)\end{array}$ & $\begin{array}{c}\mathbf{9 8} \% \\
(192 / 196)\end{array}$ & $\begin{array}{c}\mathbf{9 7 \%} \\
(190 / 196)\end{array}$ & $\begin{array}{c}\mathbf{9 0 , 3 \%} \\
(177 / 196)\end{array}$ & $\begin{array}{l}\mathbf{7 2 , 7 \%} \\
(8 / 11)\end{array}$ & $\begin{array}{l}\mathbf{1 0 0 \%} \\
(43 / 43)\end{array}$ & \\
\hline \multirow{2}{*}{ Fisioterapeutas } & pré & $\begin{array}{l}\mathbf{8 6 , 6 \%} \\
(13 / 15)\end{array}$ & $\begin{array}{c}\mathbf{7 2 , 7 \%} \\
(112 / 154)\end{array}$ & NA & $\begin{array}{c}\mathbf{9 2 , 2} \% \\
(142 / 154)\end{array}$ & $\begin{array}{l}\mathbf{4 4 , 4 \%} \\
(16 / 36)\end{array}$ & $\begin{array}{l}\mathbf{1 0 0 \%} \\
(30 / 30)\end{array}$ & \multirow[b]{2}{*}{0,0005} \\
\hline & pós & $\begin{array}{c}\mathbf{1 0 0} \% \\
(8 / 8)\end{array}$ & $\begin{array}{c}\mathbf{9 7 , 7 \%} \\
(172 / 176)\end{array}$ & NA & $\begin{array}{c}\mathbf{8 9 , 7 \%} \\
(158 / 176)\end{array}$ & $\begin{array}{l}75 \% \\
(6 / 8)\end{array}$ & $\begin{array}{l}\mathbf{1 0 0 \%} \\
(39 / 39)\end{array}$ & \\
\hline \multirow{2}{*}{ Médicos } & pré & $\begin{array}{l}75 \% \\
(6 / 8)\end{array}$ & $\begin{array}{c}\mathbf{7 2 , 6 \%} \\
(135 / 186)\end{array}$ & $\begin{array}{c}\mathbf{5 6 , 3 \%} \\
(98 / 174)\end{array}$ & NA & NA & NA & \multirow{2}{*}{0,0029} \\
\hline & pós & $\begin{array}{c}\mathbf{3 7 , 5 \%} \\
(3 / 8)\end{array}$ & $\begin{array}{c}\mathbf{9 8 \%} \\
(192 / 196)\end{array}$ & $\begin{array}{c}\mathbf{9 7 \%} \\
(190 / 196)\end{array}$ & NA & NA & NA & \\
\hline
\end{tabular}

HM- higiene das mãos antes e após contato; CE- cabeceira elevada; SOG- sonda gástrica por via oral; CIR- circuito respirador limpo; CUFF- Cuff insuflado; TRC- Track care em boas condições. NA - não avaliado, pré: pré-intervenção educacional, pós: pós-intervenção educacional. p: significativo $<0,005$

Fonte: autores 
Comparando-se a taxa média de PAV de 49,6\%, nos três meses que antecederam o treinamento, com a dos três meses subsequentes de $17,5 \%$, verifica-se uma queda de $64,8 \%$ na frequência de pneumonia associada à ventilação (gráfico 1).

Gráfico 2 - Percentual de pneumonia associada à ventilação mecânica (VAP) em pacientes internados na Unidade de Terapia Intensiva Pediátrica do HU no ano 2013, nas fases pré e pós-intervenção educacional.

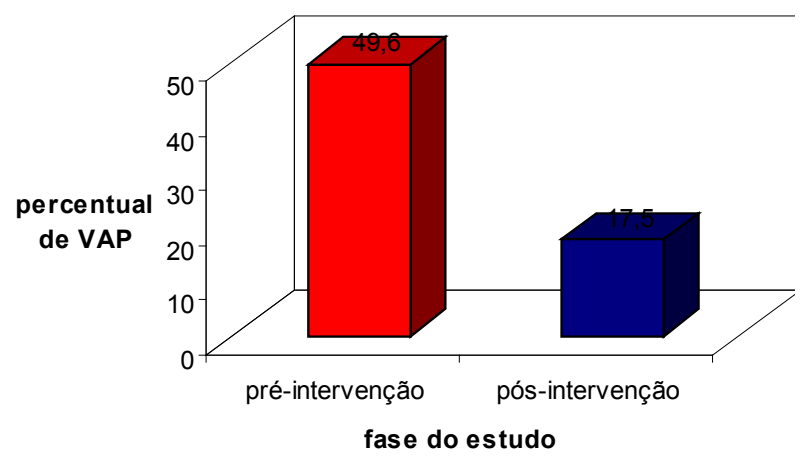

Fonte: autores

\section{Discussão}

Este estudo mostrou que a frequência de PAV diminuiu após a implantação do bundle para prevenção e que intervenções educacionais favorecem a adesão às medidas de precaução de pneumonia. Poucos estudos, até o momento, têm avaliado a efetividade destas medidas preventivas em população pediátrica. A eficácia em pacientes adultos tem sido comprovada por diversos autores, contudo os resultados não podem ser extrapolados para pacientes pediátricos.

Identificar medidas eficazes para a prevenção de PAV é tão útil quanto a aplicação correta dessas medidas na prática clínica. A união de práticas de prevenção baseada em evidências, reunidas em pacote, podem resultar em resultados melhores do que quando aplicadas individualmente.

Um hospital pediátrico americano, após implementação de uma campanha educativa intervencionista, baseada no bundle de prevenção de PAV, mostrou aumento de três vezes no tempo necessário para ocorrência de PAV, sugerindo diminuição da sua incidência (CURLEY; SCHWALENSTOCKER, 2006; FOGLIA; MEIER; ELWARD, 2007).

Muitos estudos têm mostrado uma redução nas taxas de PAV educando os profissionais de saúde sobre a epidemiologia da PAV. Bigham e colaboradores (2009), demonstraram redução na incidência de pneumonia associada a ventilação de 5,6 para 0,3 casos por 1.000 respiradores/dia após a implementação de um bundle com o objetivo de reduzir a colonização traqueobrônquica e a aspiração de secreções nos pacientes pediátricos. Neste estudo, além da diminuição da taxa de PAV houve, também, redução no tempo de internação na UTI, no tempo de ventilação mecânica e na taxa de mortalidade.

Redução na taxa de PAV foi relatada, também, num estudo multicêntrico, realizado em oito UTI pediátricas de cinco países diferentes, antes e depois de um período de intervenção educativa para sua prevenção, incluindo a aplicação do bundle. Os resultados evidenciaram redução de $31 \%$ nas taxas de PAV (ROSENTHAL et al., 2012).

Taxas de redução de PAV semelhantes (38\%) representada por uma queda de 7,9 para 4,9 casos para cada 1.000 respiradores/dia foram verificadas em outro estudo prospectivo de intervenção educacional, realizado por Babcock e colaboradores (2004) em um hospital americano. Estes mesmos autores aplicaram um programa educacional para fisioterapeutas e enfermeiros de quatro hospitais com módulos de auto estudo enfatizando medidas para melhorar as práticas de prevenção. A redução de PAV variou de 38 a $61 \%$, em três dos quatro hospitais. Em nosso estudo a diminuição de PAV verificada, em torno de $64 \%$, foi semelhante.

A influência da higiene das mãos na frequência de PAV foi avaliada em outro estudo realizado em 2007 (FOGLIA; MEIER; ELWARD, 2007) numa UTI neonatal. O aumento da higiene das mãos de $43 \%$ para 
$80 \%$ apresentou correlação com a queda de 3,35 para 1,06 casos por 1.000 respiradores/dia após período de intervenção educativa, ao contrário do nosso estudo que, apesar da redução substancial nas taxas de PAV, apresentou redução na adesão à lavagem de mãos do período pré para o, pós-intervenção.

Em um grande estudo com 415 Unidades de terapia intensiva onde avaliaram a adesão às medidas preventivas de PAV e a eficácia das mesmas na redução das taxas de PAV descobriram que o pacote era frequentemente presente, mas não bem executado. Foi necessário $95 \%$ ou mais de adesão às medidas preventivas para a redução de PAV (POGORZELSKA et al., 2011).

\section{Conclusão}

Diante da perceptível queda nas taxas de PAV em nosso serviço, acreditamos que as mudanças de atitude da equipe assistencial resultantes da intervenção educacional foram responsáveis por tal feito. Portanto, podemos concluir que a PAV pode ser prevenida pelo emprego de medidas simples e de baixo custo, quando realizadas por toda a equipe. Contudo, mais pesquisas precisam ser realizadas para definir especificamente quais medidas são realmente efetivas.

\section{Agradecimentos}

Os autores agradecem ao Conselho Nacional de Desenvolvimento Científico e Tecnológico (CNPq) pelo financiamento do presente estudo e a todos os funcionários da UTI Pediátrica do Hospital Universitário da UEL.

\section{Referências}

AGÊNCIA NACIONAL DE VIGILÂNCIA SANITÁRIA - ANVISA. Critérios diagnósticos de infecção relacionada à assistência à saúde. Brasília: ANVISA, 2013. (Série Segurança do Paciente e Qualidade em Serviços de Saúde, 2).
BABCOCK, H. M.; ZACK, J. E.; TROVILLION, E. J. M.; FRASER, V. J.; KOLLEF, M. H. An educational intervention to reduce ventilatorassociated pneumonia in a integrated health system, Chest, Chicago, v. 125, p. 2224-2231, 2004.

BIGHAM, M.T.; AMATO, R.; BONDURRANT, P.; FRIDRIKSSON, J.; KRAWCZESKI, C.D.; RAAKE, J.; RYCKMAN, S.; SCHWARTZ, S.; SHAW, J.; WELLS, D.; BRILLI, R.J. Ventilatorassociated pneumonia in the pediatric intensive care unit: Characterizing the problem and implementing a sustainable solution. Journal of Pediatrics, Saint Louis, v. 154, n. 4, p. 582-587, 2009.

CENTER FOR DISEASE CONTROL AND PREVENTION - CDC. Guidelines for preventing health-care-associated pneumonia: recommendations of $\mathrm{CDC}$ and the Healthcare Infection Control Practices Advisory Committee. MMWR, Atlanta, v. 53, p. 1-36, 2003.

CURLEY, M. A. Q.; SCHWALENSTOCKER, D. Tailoring the Institute for Health Care Improvement 100,000 Campain to pediatric settings: the example of ventilator-associated pneumonia. Pediatric Clinics of North America, Philadelphia, v. 53, p. 1231-1251, 2006.

FOGLIA, E.; MEIER, M. D.; ELWARD, A. Ventilator-associated pneumonia in pediatric intensive care unit patients. Clinical Microbiology Review, Washington, v. 20, n. 3, p. 409-425, 2007.

HORAN, T. C.; ANDRUS, M.; DUDECK, M. A. CDC/NHSN surveillance definition of healthcare associated infection and criteria for specific types of infection in the acute care setting. American Journal of Infection Control, Saint Louis, v. 36, n. 5, p. 329-332, 2008.

KOLLEF, M. H. Prevention of nosocomial pneumonia in the intensive care unit: beyond the use of bundles. Surgical Infections, Larchmont, v. 12, n. 3, p. 211-220, 2011.

LAWRENCE, P.; FULBROOK, P. The ventilator care bundle and its impact on ventilator-associated pneumonia: a review of the evidence. Nursing in Critical Care, London, v. 16, n. 5, p. 222-234, 2011. 
MORINEC, J.; IACABONI, J.; MACNETT, M. Risk factors and interventions for ventilatorassociated pneumonia in pediatric patients. Journal of Pediatric Nursing, Philadelphia, v. 27, n. 5, p. 435-442, 2012.

O'KEEFE-MCCARTHY, S.; SANTIAGO, C.; LAU, G. Ventilator-associated pneumonia bundled strategies: na evidence-based practice. Worldviews on Evidence-Based Nursing, Malden, v. 5, n. 4, p. 193-204, 2008.

POGORZELSKA, M.; STONE, P. W.; FURUYA, E. Y.; PERENCEVICH, E. N.; LARSON, E. L.; GOLDMANN, D.; DICK, A. Impact of the ventilator bundle on ventilator-associated pneumonia in intensive care unit. International Journal for Quality in Health Care, Oxford, v. 23, n. 5, p. 538-544, 2011.

PRINCIPI, N. M. D.; ESPOSITO, S. Ventilatorassociated pneumonia (VAP) in pediatric intensive care units. The Pediatric Infectious Disease Journal, Baltimore, v. 26, n. 9, p. 841-843, 2007.

ROSENTHAL, V. D.; ÁLVAREZ-MORENO, C.; VILLAMIL-GÓMEZ, W.; SINGH, S.; RAMACHANDRAN, B.; NAVOA-NG, J. A.; DUEÑAS, L.; YALCIN, A. N.; ERSOZ, G.; MENCO, A.; ARRIETA, P.; BRAN, D. E.; CASARES, A. C.; DE JESUS, M. A.; CHUCA, L.; RADHAKRISHNAN, K.; VILLANUEVA, V. D.; TOLENTINO, M. C.; TURHAN, O.; KESKIN, S.; GUMUS, E.; DURSUN, O.; KAYA, A.; KUYUCU, N. Effectiveness of a multidimensional approach to reduce ventilator-associated pneumonia in pediatric intensive care units of 5 developing countries: International Nosocomial Infection Control Consortium findings. American Journal of Infection Control, Saint Louis, v. 40, n. 6, p. 497-501, 2012. 
Recebido em: 01 ago. 2014 Aceito em: 02 out. 2014. 